\title{
Accelerated theta-burst repetitive transcranial magnetic stimulation for depression in South Africa: A series of nine cases
}

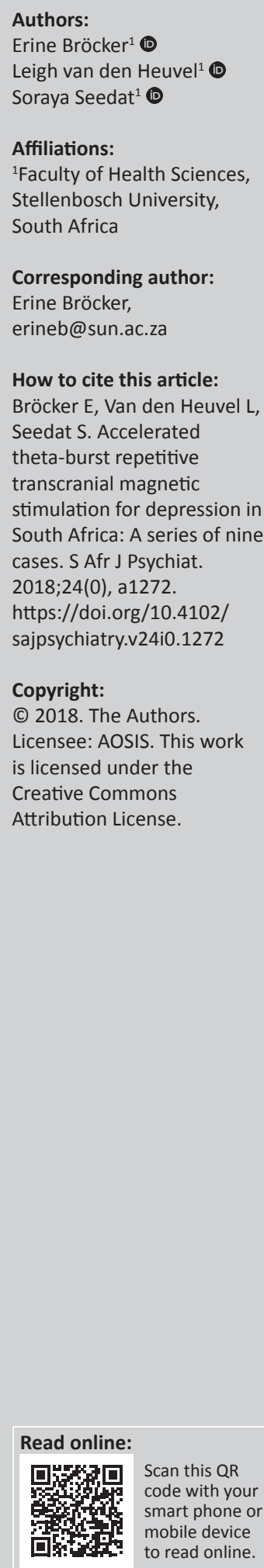

Introduction: This case series documents local experience using accelerated theta-burst repetitive transcranial magnetic stimulation (rTMS) as a supplementary treatment for depression in both major depressive disorder (MDD) and bipolar disorder (BD).

Methods: Nine consenting patients (MDD $=7$; BD [major depressive episode] $=2$ ) received the accelerated theta-burst protocol consisting of three magnetic pulses delivered $20 \mathrm{~ms}$ apart and repeatedly delivered every $200 \mathrm{~ms}$, resulting in a $5 \mathrm{~Hz}$ theta rhythm over the left dorsolateral prefrontal cortex (DLPFC). Treatment comprised 20 sessions delivered over 8 days. Accelerated theta burst rTMS treatment provides more stimuli over a shorter period of time, thus potentially increasing feasibility and cost-effectiveness. Improvement was monitored using the Centre for Epidemiological Studies Depression (CES-D) scale and the Clinical Global Impression (CGI) scale at baseline, day 5 and day 8 of rTMS treatment. All patients remained on their prescribed medication for the duration of rTMS treatment. We performed a Wilcoxon matched-pairs signed rank test to determine whether there was a difference in scores from baseline to post-treatment. The CES-D scores decreased significantly from baseline (Mdn 38.0; IQR 31.0; 51.0) to posttreatment (Mdn 17.0; IQR 11.0; 28.5; $Z=-2.55, p=0.011$ ). The CGI severity scores also decreased significantly between baseline (Mdn 4.0; IQR 4.0; 5.0) and post-treatment (Mdn 3.0; IQR $3.0 ; 4.0 ; Z=-2.43, p=0.015)$.

Results: Five patients demonstrated at least a 50\% symptom reduction on the CES-D scale. The most commonly reported adverse effect was mild headache, which lasted a few hours during and post-rTMS treatment.

Conclusion: A limitation of these findings is that this was a small case series without a control arm; however, the findings suggest that the accelerated theta burst rTMS protocol for depression was well tolerated with most patients also experiencing symptomatic improvement by day 8 .

Note: A selected abstract from papers presented at the 19th National Congress of the South African Society of Psychiatrists in 'Professional Psychiatric Practice: Medical, Socio-Economic \& Cultural Perspectives', 21-24 September 2018, at the CSIR, Pretoria, South Africa. The congress is hosted by South African Society of Psychiatrists (SASOP).

This work is supported by the FMHS Strategic Equipment Fund and the South African Research Chairs Initiative in post-traumatic stress disorder (PTSD), funded by the Department of Science (DST) and Technology and National Research Foundation (NRF). 